Questions vives

\section{Questions Vives}

Recherches en éducation

$N^{\circ} 27 \mid 2017$

(Re) découvrir John Dewey : quelle actualité en formation des adultes?

\title{
Enquête inter-objective, environnement " augmenté » et développement professionnel
}

Collaborative Inquiry, Video-enhanced Learning Environment and Teacher Professional Development

Alain Muller et Valérie Lussi Borer

\section{OpenEdition}

Journals

\section{Édition électronique}

URL : http://journals.openedition.org/questionsvives/2097

DOI : 10.4000/questionsvives.2097

ISSN : 1775-433X

\section{Éditeur}

Université Aix-Marseille (AMU)

\section{Édition imprimée}

Date de publication : 31 juillet 2017

ISBN : 978-2-912643-51-3

ISSN : 1635-4079

Référence électronique

Alain Muller et Valérie Lussi Borer, « Enquête inter-objective, environnement « augmenté » et développement professionnel », Questions Vives [En ligne], N 27 | 2017, mis en ligne le 31 décembre 2017, consulté le 19 avril 2019. URL : http://journals.openedition.org/questionsvives/2097 ; DOI : 10.4000 /questionsvives.2097

Ce document a été généré automatiquement le 19 avril 2019.

\section{(c) (1) (9)}

Questions Vives est mis à disposition selon les termes de la licence Creative Commons Attribution Pas d'Utilisation Commerciale - Pas de Modification 4.0 International. 


\title{
Enquête inter-objective, environnement « augmenté » et développement professionnel
}

\author{
Collaborative Inquiry, Video-enhanced Learning Environment and Teacher \\ Professional Development
}

Alain Muller et Valérie Lussi Borer

\section{Introduction}

1 Les différents apports de Dewey concernant les conceptions de l'enquête, du développement humain et des transactions entre organisme et environnement nous apparaissent particulièrement fertiles pour concevoir et mettre en œuvre des dispositifs innovants de formation initiale et continue à l'enseignement. L'usage que nous faisons du pragmatisme de Dewey a déjà été présenté dans plusieurs de nos articles, ceci sous divers aspects : processus de transformation de l'activité suscités par une enquête (Lussi Borer \& Muller, 2013; 2014a ; 2014b), mise en œuvre d'une enquête collaborative (Lussi Borer \& Muller, 2016; Muller, 2014), articulation de l'activité des formateurs et des formés dans une enquête (Muller \& Lussi Borer, 2017). Cet article qui procède d'une réflexion conceptuelle illustrée d'exemples issus de nos recherches empiriques, s'intéresse plus particulièrement à décrire les processus de transformation des relations sujetenvironnement suscités par des enquêtes collaboratives soutenues par un environnement « augmenté » de traces vidéo de l'activité. De tels environnements nous apparaissent propices à enrichir les expériences vécues par les participants à l'enquête et à susciter des transformations de l'activité dans une perspective de développement professionnel.

2 Nous présentons 1) la manière dont nous reprenons les concepts d'enquête, enquête sociale et inter-objective, 2) ce que nous entendons par environnement " augmenté », 3) les processus de transformation potentiels liés à ce type d'environnement, 4) les principes nécessaires pour que de tels dispositifs de formation soutiennent le développement 
professionnel, 5) les dimensions temporelles, objectales et relationnelles propres à l'enquête collaborative, 6) la rationalité pratique et située impliquée par ce type d'enquête.

3 Un ensemble d'exemples empiriques présentés sous forme d'encadrés jalonnent le texte. Ils visent à permettre au lecteur soit de s'y arrêter au fil de sa lecture pour ancrer dans le concret la réflexion conceptuelle que nous développons, soit d'entreprendre une lecture linéaire de cette réflexion pour revenir dans un second temps aux exemples.

\section{Enquête, enquête sociale, enquête inter-objective}

4 Pour Dewey $(1993 ; 2010)$ la relation d'un organisme et de son environnement est une transaction: l'organisme et l'environnement sont profondément intégrés l'un à l'autre, ils collaborent à une seule et même activité, ils ne sont pas ontologiquement détachés l'un de l'autre. La rupture de cette intégration, l'irruption d'un déséquilibre dans la relation organisme-environnement, génère un processus de recherche d'un nouvel équilibre, d'une nouvelle intégration. C'est ce processus que, de manière générique, Dewey nomme enquête. Celle-ci se déploie en trois moments. Premier moment, la situation - autre nom pour l'interaction de l'organisme et de son environnement - est indéterminée, c'est-à-dire trouble, incertaine, opaque. Second moment, ce qui pose problème, ce qui fait le déséquilibre est identifié: on est alors dans une situation problématique. Troisième moment, une solution est trouvée au problème et, par là, la situation (re)devient déterminée. Mais l'équilibre retrouvé n'est pas tout à fait le même que celui « de départ »: à travers l'enquête, et parce qu'ils sont ontologiquement solidaires, organisme et environnement se sont mutuellement transformés. Mais ce nouvel équilibre n'est bien sûr que provisoire, d'autres situations indéterminées vont se présenter, appelant de nouvelles enquêtes, de nouveaux types d'intégration organisme-environnement, et ainsi de suite dans un processus continu et infini de développement. Ajoutons que ce schème, dans sa généricité, est le même pour toutes les sortes d'enquêtes, de la recherche de nourriture à la résolution d'un problème de physique théorique, ou d'un problème social.

5 Car, pour Dewey, l'homme est un organisme social de part en part, c'est-à-dire en transaction permanente avec un environnement social. Par là, il est inévitablement entrainé à entrer dans des enquêtes sociales. Et ceci parce que les conditions sociales de vie ne sont pas souvent favorables au développement des individus - ou au moins de certains individus - et qu'à l'inverse les comportements des individus ne sont pas toujours orientés vers le développement du collectif. La fonction d'une enquête sociale est ainsi

«...d'apporter en l'absence de moyens de remédiation immédiatement disponibles, les objets et méthodes qui permettent aux individus, pluriels ou singuliers, associés ou isolés, liés à la tradition ou à l'institution, de réorganiser leur environnement lorsque les interactions avec ce dernier sont perturbées et ne leur apportent plus les éléments dont ils ont besoin pour développer leurs activités et leur personnalité dans la voie qui est la leur » (Zask, 2015, pp. 71-72).

Prise sous un autre angle, on peut dire que l'enquête sociale vise à constituer un collectif en communauté:

"L'activité en association n'a pas besoin d'explication; les choses sont faites de cette manière. Mais jamais un degré quelconque d'action collective et agrégée ne constitue par lui-même une communauté. Pour des êtres qui observent et pensent, et dont les idées sont absorbées par des impulsions et deviennent des sentiments et 
des intérêts, "nous" est aussi inévitable que "je”. Mais "nous" et "nôtre” n’existent que quand les conséquences de l'action combinée sont perçues et deviennent un objet de désir et d'effort, de même que "je" et "mien" n'apparaissent sur la scène que quand une part distinctive dans l'action mutuelle est consciemment affirmée et revendiquée » (Dewey, 2010, pp. 246-247).

7 L'enquête sociale est donc une enquête collaborative au sens où plusieurs individus (singuliers ou collectifs) travaillent ensemble à transformer leurs situations respectives. Mais, dans la mesure où les individus impliqués dans l'enquête ne partagent pas forcément les mêmes intérêts, n'occupent pas les mêmes "places », leur collaboration réelle - leur constitution en communauté - suppose un processus d'inter-objectivation, soit « la constitution d'objets en fonction des possibilités d'accord entre expériences plurielles»(Zask, 2004, p. 148). Cet accord ne signifie pas que les divers individus impliqués dans l'enquête travailleront sur le même objet et en auront une conception identique, mais qu'ils travailleront sur des objets leur permettant mutuellement de transformer leur activité. Ces objets d'enquête, sortes d'interfaces entre individus, doivent donc être co-construits pour qu'ils soient justement reconnus par chacun comme étant le « nôtre » et comme étant le « mien ».

Un exemple de passage du collectif professionnel à la communauté professionnelle

Le passage du simple collectif à la communauté professionnelle semble être fortement solidaire de la production d'un (ou de plusieurs) objets inter-objectifs. Dans une des trois écoles que nous avons suivies dans le cadre du projet Plus de maitres que de classes, cette construction d'une communauté s'est déroulée en trois moments.

Lors de la première année, les enseignants se sont surtout montrés intéressés à avoir accès aux "modes de travail à deux» de leurs autres collègues. Leur objet de recherche était assez général : comment travailler à deux dans une classe, comment se partager la parole, comment se partager l'espace... ? On pourrait dire que cet objet flou répondait à un accord minimal entre personnes, correspondant à l'intérêt «cumulé » d'une série de “je" désireux "d'en savoir un peu plus». Série de "je" plus que de "nous" au sens où cet intérêt large pour l'activité des autres semblait être porteuse d'objets de préoccupations divers et singuliers.

La seconde année cet intérêt pour le travail à deux dans la classe s'est prolongé, mais en se focalisant de manière plus ciblée sur l'activité elle-même et concrétisé dans un petit noyau de "sous-objets" d'enquête : s'auto-contrôler (intensité de la voix par exemple) pour ne pas déranger l'autre, malentendus, double attention collègue-élèves... À cette élaboration d'un objet-activité (et de ses composantes) vraiment commun a correspondu la constitution progressive d'une communauté, d'un "nous", au sens où tous les enseignants étaient sur le même objet.

La troisième année a vu croître le travail collectif. L'objet d'enquête, toujours l'activité du travail à deux, mais vue maintenant dans son rapport à l'activité des élèves, a suscité l'élaboration et la mise en œuvre de modes pluriels et diversifiés de co-intervention dans le cadre de conceptions de séquences communes par niveaux d'enseignement. Bref, le "nous" a continué à se développer à travers l'affirmation des “je” et de leurs spécificités.

\section{Environnement « augmenté »}

8 Comprendre ce que nous entendons par environnement " augmenté » nécessite d'abord de préciser ce qu'est un environnement ordinaire pour Dewey, ainsi que de décrire sa conception du développement de l'expérience. 
9 L'environnement n'est pas le monde, ni un milieu « objectif », mais cette partie du monde ou d'un milieu avec lequel un organisme est en transaction, soit cette partie du monde ou d'un milieu qui participe à son maintien en vie et à son développement : l'environnement est «la somme des conditions qui entrent de manière active dans la direction des fonctions de n'importe quel être vivant » (Dewey, 1911, p. 438). On pourrait dire aussi, que l'environnement d'un organisme est ce qui, dans le monde, est significatif pour lui, non pas au sens où cet organisme ne saisirait le monde qu'à l'aune des projections de sa subjectivité, mais au sens où ce qui fait signe pour lui est cette partie du monde avec laquelle il est en transaction. ${ }^{1}$ Comme on l'a vu, les processus d'enquête ne consistent pas seulement à retrouver un équilibre momentanément rompu, mais aboutissent, à travers le réajustement de la relation, à une transformation et de l'organisme et de l'environnement. Cette transformation correspond du côté de l'organisme à la production de nouvelles habitudes et/ou de nouvelles connaissances; du côté de l'environnement à son "élargissement», au sens où, à travers la suite ininterrompue d'enquêtes, l'expérience se fait plus complète, les relations se complexifient, se diversifient, se multiplient.

De manière très générale, créer un environnement "augmenté » dans le cadre d'un dispositif visant le développement professionnel consiste à rendre, artificiellement et volontairement au moins possible si ce n'est probable, une multiplication et/ou une diversification des interactions à l'environnement ordinaire, en postulant que, par làmême, les relations à cet environnement vont se transformer.

11 Une des manières les plus classiques d'augmentation de l'environnement ordinaire consiste dans un premier temps à recueillir des traces de l'activité : enregistrements audio ou vidéo, photographies, notes ethnographiques, traces de travaux d'élèves, etc. Les participants à l'enquête sont ensuite confrontés, individuellement ou collectivement, à ces traces à travers des dispositifs de formation impliquant des autoconfrontations simples, croisées, ou encore collectives.

12 Ces traces de l'activité enrichissent l'environnement ordinaire au sens où elles «contiennent» et permettent de voir et d'entendre ce qui, dans le cadre du travail quotidien, ne se voit et ne s'entend pas, ou très difficilement et rarement.

Mais l'environnement ordinaire bénéficie aussi d'une sorte "d'augmentation sociale», dans la mesure où le travail collectif sur les traces d'activité et les échanges à leur propos permettent de multiplier les points de vues, les interprétations divergentes, les «croisements » d'idées, etc. Bref, les traces et le travail sur ces traces consistent bien à multiplier et diversifier les interactions à l'environnement ordinaire, permettant par làmême la découverte ou la mise en lumière de dimensions cachées ou tacites, ainsi d'élargir l'espace de ce qui fait sens dans le travail quotidien. 
Quelques exemples d'enrichissement de l'environnement ordinaire à travers le recueil de traces de l'activité

La gamme de possibilités d'enrichissement de l'environnement à travers le recueil de traces de l'activité est quasi infinie. Voici quelques exemples issus de nos différentes interventions en formation initiale ou continue à l'enseignement.

Voir l'activité de l'enseignant ou des élèves sous plusieurs angles et avec différents zooms, ce qui rend par exemple possible, dans le cas d'une prise de vue en fond de classe, de voir ce que voient les élèves habituellement.

Voir des situations de même type (même contenu, même format pédagogique...), ainsi repérer du même et du différent.

Voir sa propre activité d'enseignant, soit de manière "objective- analytique ", soit de manière plus personnelle, c'est-à-dire en retrouvant des pensées, des perceptions, des émotions, etc., vécues en situation.

Voir l'activité de ses collègues soit de manière "objective-analytique », soit de manière plus personnelle, c'est-à-dire en s'y projetant comme si c'était sa propre activité.

Voir l'activité des élèves dans sa continuité temporelle : par exemple, suivi d'un élève ou d'une paire d'élèves dans leur résolution d'une tâche.

Plus vaguement repérer des «ambiances", des "climats ", des " atmosphères »... bref, les aspects qualitatifs et fugitifs des situations, souvent peu saisissables quand on est en action et focalisé sur la gestion d'événements.

\section{Enquête inter-objective sur un environnement " augmenté » et transformation des activités}

\subsection{Une « augmentation » qualitative}

14 Mais «l'augmentation » de l'environnement n'est pas que quantitative - comme pourrait le laisser penser le lexique utilisé : multiplication, diversification... - elle est surtout qualitative au sens où, à travers la confrontation collective à des traces de l'activité (de soi, des autres), les transactions à l'environnement ordinaire elles-mêmes vont être transformées, ouvrant en cela un espace possible de transformation réelle de l'activité. Cette "augmentation qualitative" prend sa source, de manière générale, dans les processus phénoménologiques et sémiotiques que le visionnement de films (de soi ou d'un autre) tend à susciter ${ }^{2}$ :

- engagement mimétique, soit voir ce que fait un autre comme si c'était soi-même qui le faisait ;

- dégagement «allo-centré », soit voir ce qu'on fait soi-même comme si c'était un autre qui le faisait ;

- expérience fictionnelle, soit pouvoir imaginer des faire autrement ce qui est vu ;

- actualisation d'insatisfaction, soit comprendre, en regardant un autre travailler, sur quoi repose le sentiment d'insatisfaction trouble relatif à sa propre activité (ainsi être en mesure de la modifier) ; 
- production d'insatisfaction, soit prendre conscience, en regardant un autre travailler, que ce que l'on pensait être satisfaisant dans son activité propre ne l'est pas tant que ça (ainsi être en mesure de modifier cette dernière) ;

- multiplication des niveaux de « saisie » de l'activité et de l'interaction entre ces niveaux, soit ne pas rester uniquement dans un registre évaluatif (registre le plus souvent « spontanément » mobilisé dans un premier temps), mais aussi décrire, interpréter, expliquer, comparer, confronter...

Comprendre ce qui rend possible «l'augmentation qualitative» de l'environnement et quelles sont ses conséquences transformatives sur les activités concrètes suppose de saisir le déploiement de ce type de processus dans le cadre d'une enquête inter-objective. Nous allons donc essayer de comprendre ce qui se passe quand une personne enquête sur son activité filmée en collaboration avec d'autres personnes.

\subsection{Méthode}

Précisons premièrement que notre démarche est en même temps descriptive - elle vise à décrire ce qu'il en est de la relation sujet-environnement dans ce type de situation - mais aussi normative au sens où nous décrivons ce qu'il en est de cette situation quand elle est véritablement inter-objective et recèle le plus haut degré de "potentiel transformatif ". Afin de mieux faire comprendre par comparaison ce qu'est véritablement cette situation, nous décrivons aussi ce qu'elle n'est pas, soit des situations empiriquement possibles, mais ne correspondant pas à une situation à haut degré de "potentiel transformatif » et véritablement inter-objective.

Deuxièmement, notre formalisation des relations sujet-environnement repose sur la phanéroscopie de Peirce (1978) et principalement sur la distinction entre relation dyadique et relation triadique. Très succinctement, une relation dyadique est une relation directe entre deux éléments "qui se font face ", «s'opposent ", «se confrontent ». Une relation triadique est une relation dans laquelle un élément met en relation deux autres éléments et joue un rôle de médiateur entre ces éléments.

Schéma 1

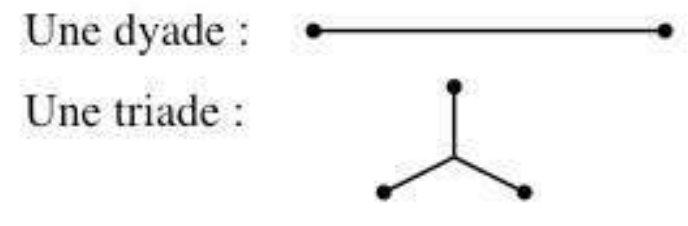

19 Troisièmement, nous menons cette entreprise pas à pas, de la situation la plus « simple » à la situation la plus « complexe », soit :

1. un sujet confronté seul aux traces filmées de son activité ;

2. un sujet confronté aux traces de son activité filmée en compagnie d'un autre sujet (chercheur, formateur, chercheur-formateur), soit une situation d'autoconfrontation simple ;

3. un sujet confronté aux traces de son activité en compagnie d'un autre sujet (ou d'autres sujets) confronté(s) aux traces de son (leurs) activité(s), soit une situation d'autoconfrontation croisée ou collective. 


\section{3. Être confronté aux traces de sa propre activité} particulière ne consiste pas seulement en une objectivation de ce qui a été vécu (cf. schéma 2), comme si la situation visionnée n'était plus celle de celui qui visionne. Car, à travers le visionnement, la situation de classe est, en même temps qu'elle est objectivée, revécue de l'intérieur : le film fait «remonter » les émotions, les pensées fugitives, les choix opérés, les renoncements, etc. Bref, il y a «engagement» dans la situation originelle autant que «dégagement » de celle-ci, le sujet est "dedans » comme il est « dehors » ou, pourrait-on dire, il est dans une sorte de « hors-dedans » (cf. schéma 3).

Schéma 2

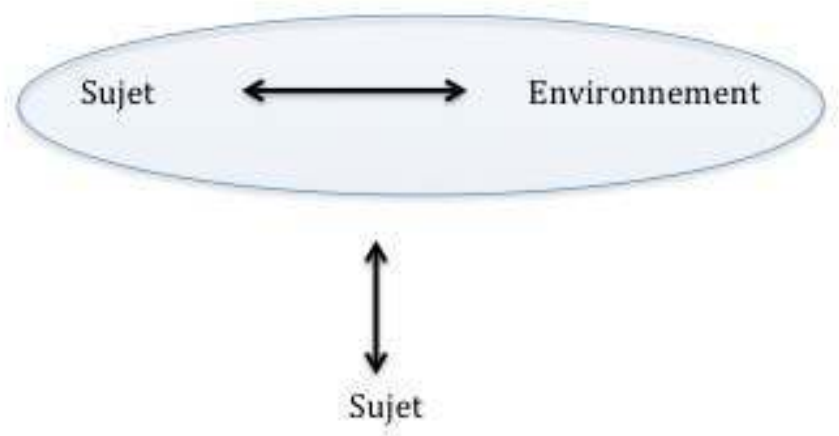

Schéma 3

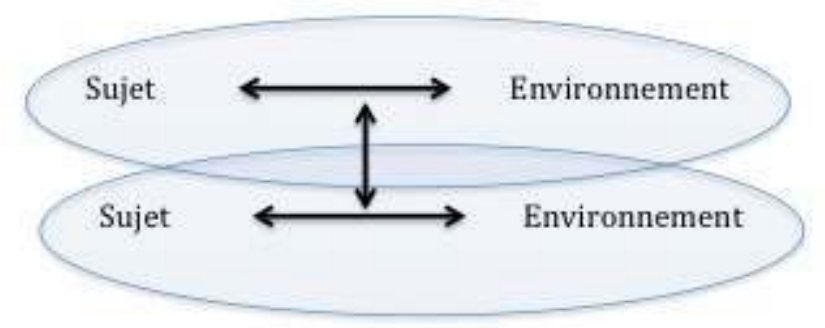

23

L'objectivation de la situation de classe dans la situation d'autoconfrontation ne procède ainsi pas d'une «sortie » de celle-ci - comme si on la mettait à distance - mais de sa « remise en jeu ». C'est à notre sens dans cette «dualité existentielle » de la situation d'autoconfrontation que recèle son potentiel de développement professionnel. 
24 Un sujet ne faisant que contempler et analyser de l'extérieur sa situation de classe pourrait en tirer quelques «leçons», mais celles-ci resteraient de l'ordre de considérations « intellectuelles». Il pourrait certes accéder à des pans inconnus de son activité et de celle de ses élèves, voir des événements qu'il n'avait pas remarqués, « prendre conscience » qu'il n'a pas fait exactement ce qu'il pensait avoir fait, etc. Mais ces nouveaux faits émergeant de son enquête objectivante resteraient comme détachés des faits ou des événements vécus en classe. La transformation de ceux-ci, la transformation de son activité ne seraient possibles encore une fois que d'un point de vue « intellectuel», de l'ordre d'une auto-prescription extérieure à la logique interne qui anime son activité en classe :j'ai fait comme ci...j'aurais dû faire (je ferai) comme cela.

À l'inverse, uniquement revivre (sous forme de souvenirs, de réminiscences, de « ruminations ») la situation ne consisterait qu'à répéter celle-ci, à y retrouver les mêmes faits, les mêmes événements, sans évidemment pouvoir transformer ceux-ci.

Par contre « revivre extérieurement » la situation de classe, s'y « ré-engager » de manière « dégagée » permet de faire émerger de nouveaux faits, d'accéder à des pans inconnus de son activité, mais ceci en lien avec les faits et événements vécus en classe. C'est ce qui a été $v u$ en classe qui est maintenant vu autrement, sous un nouveau jour, qui prend une nouvelle signification. C'est ce qui a été vécu en classe, qui est maintenant revécu autrement, qui prend une nouvelle signification. Bref, ce ne sont pas de nouveaux objets d'enquête qui apparaissent, mais les objets de l'enquête - avec leur concrétude, leur logique interne - qui ré-apparaissent sous une nouvelle apparence, qui sont transformés. Ainsi, les transformations futures de son activité ne sont plus projetées sous forme d'auto-prescriptions extérieures à celle-ci, mais sous forme d'une réalisation de ce qui existe déjà dans l'activité visionnée, d'une réalisation de ses potentialités : j'ai fait comme ci... et dans ce comme ci, il y avait (aussi... déjà...) du comme ça... que j'aurais pu faire...

Ainsi, plutôt que de dire comme plus haut qu'on a affaire à un processus d'enquête sur les processus d'enquête menés en classe, il semble plus juste de dire qu'on a affaire à un processus d'enquête dans les processus d'enquête menés en classe. 


\subsection{L'inter-objectivation de sa propre activité} que le sujet $b$ est en relation à son objet (qui est un autre objet que celui du sujet $a$ ), et que sujet $\mathrm{a}$ et sujet $\mathrm{b}$ échangent à propos de leurs objets respectifs : il n'y a donc pas d'objet co-construit, d'enquête inter-objective. Dans une telle situation, tout ce que le sujet $b$ peut apporter au sujet a est un point de vue extérieur au sien, peut-être intéressant, mais qui ne peut pas prendre à sa charge le point de vue du sujet a, ni apporter son point de vue autre dans le point de vue du sujet a. La transformation de l'activité du sujet a, ne serait 
possible, encore une fois, que comme prescription extérieure à la logique interne qui anime son activité en classe : tu as fait comme ci... tu aurais dû faire (j'aurais fait) comme cela.

À notre sens, parler d'enquête inter-objective présuppose que le sujet b opère comme élément médiateur, soit comme le troisième terme, d'une seule et unique relation à trois termes (cf. schéma 5).

Schéma 5

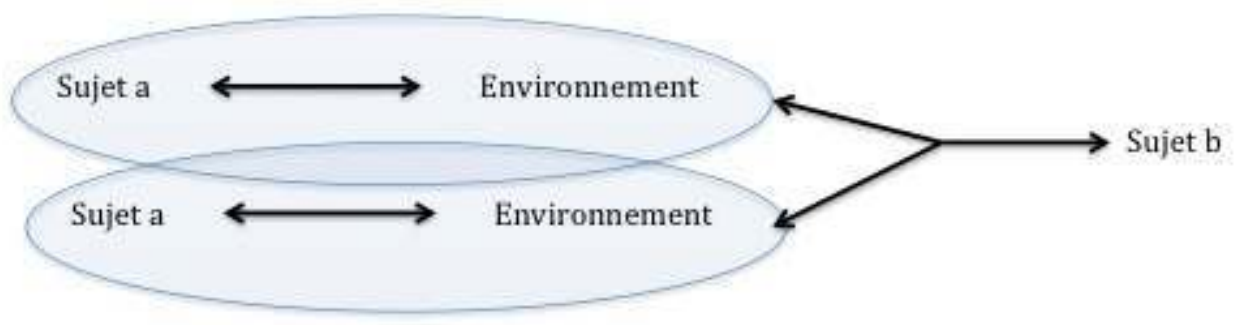

Le sujet a est en relation à sa situation de classe par la médiation du point de vue du sujet $b$. Dans l'autre sens, c'est à travers le point de vue du sujet a que le sujet b entre en relation à la situation de classe visionnée. Ils partagent un même objet, et si leurs points de vues sur celui-ci peuvent diverger, cette divergence se fait en intégrant le point de vue du coenquêteur. Bref, ce qu'apporte le sujet b au sujet a est un point de vue " autre- intérieur ", un point de vue dans le point de vue du sujet a. La transformation de l'activité du sujet a devient alors possible comme transformation depuis elle-même, comme réalisation de son propre potentiel, celui-ci étant "révélé " par le sujet b. On a affaire donc à un " moiautre » s'exprimant à deux voix conjointes : j'ai-tu as fait comme ci... et dans ce comme ci, il y avait (aussi... déjà...) du comme ça... que je-tu aurais pu faire... ${ }^{3}$

Cette situation se complexifie encore dans les cas d'autoconfrontation croisée, dans la mesure où le sujet $b$ entre en médiation dans la relation sujet $a-$ situation de classe comme un autre sujet avec sa propre situation de classe (cf. schéma 6).

\section{Schéma 6}

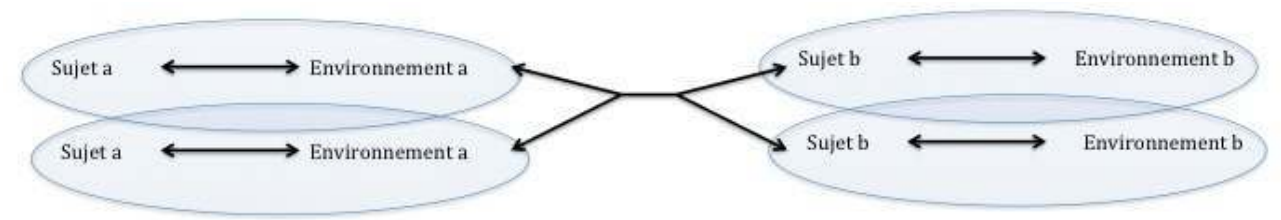

37 Ce qu'ajoute cette situation à celle décrite plus haut, c'est que le point de vue " autreintérieur » apporté par le sujet b dans le point de vue du sujet a est «porteur» de sa propre situation de classe, qu'ainsi ce point de vue est aussi celui d'une autre activité, ce qui augmente encore l'espace des significations et des transformations possibles de l'activité du sujet a, nouvel espace qu'on pourrait nommer « même - différent ». Eu égard à la structure triadique de la relation, cette autre activité du sujet $b$, ne peut exercer son altérité que dans la mesure où elle est aussi et en même temps celle propre du sujet a (et il en est de même du point de vue - activité du sujet a dans celui du sujet b). ${ }^{4}$

Quant aux autoconfrontations collectives, elles accroissent encore plus l'espace des médiations, ainsi multiplient les significations et transformations possibles de l'activité des sujets $a, b, c, d . .$. 
Un exemple de passage d'une transaction où chaque sujet garde son point de vue (schéma 4) à une transaction productrice d'un point de vue intégré, d'un espace " moi - autre » (schéma 5)

Lors de l'autoconfrontation croisée avec une autre enseignante en formation, l'enseignante revient sur le vécu du moment où elle interrompt le travail en duo et s'exprime ainsi : C'est vrai que je regrette un peu parce que c'est un petit dialogue, ça ne prend pas longtemps et je les reprends deux fois. Je les reprends une fois pour la consigne et là je les reprends pour le bruit parce que j'avais l'impression... Je ne sais pas si ça se voit dans le film ou si c'est mon imagination, c'est mon gros problème. Et moi j'avais l'impression que vraiment c'était trop bruyant alors je les reprends encore une fois.

La deuxième enseignante réagit: Non on n'a pas vraiment... J'ai l'impression que c'est une bénédiction moi (rires) et qu'ils sont tous dans leur dialogue en allemand. C'est peut-être simplement ça... Et elle suggère de prendre comme critère plutôt le fait que les élèves parlent en allemand que de tenter de définir un niveau sonore «standard».

La première enseignante développe alors une réflexion sur sa sensibilité au niveau sonore : Mais c'est très très... Pour moi c'est très fort. C'est vraiment des moments où je commence à stresser de me dire... je pense que c'est des choses bêtes, de me dire si quelqu'un passe dans les couloirs et nous on fait un de ces brouhahas... Des fois j'entends à côté des portes et je vois comment font les autres parce que je veux savoir quel est le taux de volume...

En creusant la question de l'effet du niveau sonore sur ses émotions (stress, peur), elle identifie la peur du regard extérieur qui l'habite et qui prend une place telle qu'elle l'empêche de prendre en compte et d'apprécier l'activité des élèves. Cette identification émerge en réponse au point de vue contrasté de la deuxième enseignante. Mais le point de vue de la deuxième enseignante reste encore "extérieur " au point de vue de la première. Elles sont chacune sur leur propre objet: "peur du bruit » pour l'une, "appréciation de l'activité des élèves » pour l'autre. Plus tard, la première enseignante revient sur la peur qu'elle éprouve quand le niveau sonore monte: C'est peut-être une peur que j'ai qui vient de l'année passée ou je sais pas C'est pas que j'aie peur qu'ils ne soient pas tous en train de travailler, j'ai peur de les perdre, c'est toujours la même peur. [...] Je sais pas si à ce moment c'est vraiment justifié, mais...

La deuxième enseignante approuve : Je crois que ça fait peur de laisser les élèves travailler par deux ou en groupe de manière autonome puis après de devoir les reprendre, c'est stressant. C'est d'ailleurs pour cela que certains ne le font pas, j'ai pas vu dans tous les cours d'allemand du travail entre partenaires.

La première conclut : Mais je pense que c'est vraiment une histoire de... maintenant si j’y réfléchis, une histoire de regard extérieur. J'ai peur que quelqu'un de l'extérieur passe... et c'est ça, la peur... comme quand je suis en ville avec ma fille et je dois l'engueuler et j'ai peur des regards c'est la même... je pense que c'est le même stress...

On voit qu'en échangeant, l'enseignante explicite la peur et le stress qui conditionnent son comportement et qu'elle identifie mieux : peur de perdre le contrôle sur les élèves et peur face aux attentes extérieures (peur du regard sur sa légitimité d'enseignante, de mère). L'enquête menée sur son activité par le double mouvement « hors-dedans » et par l'apport d'un point de vue différent par la deuxième enseignante qui convoque des dimensions positives de l'activité des élèves pour nourrir l'enquête (les élèves parlent allemand, sont dans le dialogue) permet à la première de mieux comprendre la dynamique de son activité.

Ce qu'elle vivait comme relevant d'une contradiction personnelle est réélaboré comme un conflit entre ses propres attentes et celles (plus ou moins imaginaires) de l'extérieur (collègues, hiérarchie...). Ce nouveau sens donné à son activité, et qui lui ouvre un espace potentiel de transformation de celle-ci, émerge de l'intégration du point de vue de la deuxième enseignante dans le sien (ce que l'activité des élèves recèle de positif). Mais cette intégration est rendue possible parce que, de son côté, la deuxième enseignante intègre elle-même le point de vue de la première (Je crois que ça fait peur de laisser les élèves travailler par deux...) et prend en compte son stress. 


\subsection{Trois espaces de nouvelles significations en interaction}

\section{objective ouvrent un jeu d'interactions dans trois espaces de nouvelles significations. ${ }^{5}$}

Il y a bien ici « augmentation qualitative » de l'environnement dans la mesure où, comme on l'a vu, le «dehors » ne fait pas que s'ajouter au «dedans », «l'autre » ne fait pas que s'ajouter au «moi », le «différent» ne fait pas que s'ajouter au "même», mais qu'à travers leur respectives interpénétrations sont créées de nouvelles interactions à l'environnement. On peut faire l'hypothèse raisonnable ${ }^{6}$ qu'il en est de même de la relation de ces trois espaces de nouvelles significations : ils ne font pas que s'additionner, mais, dans une logique similaire à leur propre production, ils tendent à s'interpénétrer, tel ou tel espace jouant le rôle de médiation des deux autres espaces (cf. schéma 6). S'ouvre par là un espace de nouvelles significations, de nouvelles interactions à l'environnement, « au carré».

\section{Dispositif}

Il ne suffit pas de produire un environnement « augmenté », soit de recueillir des traces de l'activité et de l'expérience et de faire travailler un collectif sur celles-ci pour que s'ouvrent les trois espaces de nouvelles significations « dedans - dehors ", « moi - autre » et «même - différent ». Produire du développement professionnel, suppose que l'activité qui se déploie au sein d'un environnement « augmenté » soit tenue le plus possible par un certain nombre de principes.

Ces principes sont évidemment en lien avec la conception pragmatiste du développement de Dewey.

1. Les objectifs d'une formation professionnelle ou d'une intervention doivent être "dégagés " de l'intérieur de l'activité, afin de pouvoir en exprimer les potentialités de développement, et non être définis en extériorité, du point de vue des prescriptions institutionnelles: un « vrai » objectif est « la prévision de diverses conséquences découlant de différentes façons 
d'agir dans une situation donnée et l'utilisation de cette prévision pour diriger l'observation et l'expérimentation » (Dewey, 1990, p. 160).

2. L'objet central de formation ou d'intervention n'est pas le travail prescrit mais le travail réel, ce qui implique d'avoir accès à ce dernier à travers le recueil de traces de l'activité.

3. Les transformations des activités s'appuient ainsi sur des potentiels repérés dans l'activité existante mais qui ne sont pas encore actualisés et non pas sur leur distance à un étalon (ce qui devrait être).

4. L'enquête est menée sur les activités et non pas sur les personnes menant ces activités.

5. Les formateurs ou les accompagnateurs, tout comme les praticiens participant à l'enquête, adoptent une posture respectueuse, bienveillante et empathique les uns envers les autres, ce qui n'interdit pas la critique.

Ces principes, dont les formateurs-accompagnateurs sont les garants, doivent être mis en œuvre dans toutes les étapes de l'enquête, mais surtout être " explicités » et " explorés » dès les premiers temps de celle-ci. Il s'agit par exemple que les personnes engagées dans l'enquête s'approprient quelques concepts issus du champ de l'analyse du travail, afin de comprendre dans quelle optique le travail de retour sur les traces d'activité va être mené. Il s'agit, autre exemple, de susciter une "ouverture» du regard très souvent «spontanément » uniquement normatif sur l'activité, de développer des compétences diversifiées de description et d'interprétation de ce qui est vu.

Un exemple de dispositif permettant le développement de compétences diversifiées de compréhension de l'activité

Cette entreprise est souvent initiée en confrontant les participants engagés dans l'enquête à des extraits filmés d'activités d'enseignement, puis en recueillant leurs réactions " spontanées ». La plupart du temps, ces réactions sont de l'ordre de jugements plus ou moins argumentés. Il s'agit alors de travailler à ouvrir ce regard uniquement normatif, en obligeant les participants d'une part à décrire ce qu'ils voient de la manière la plus neutre possible - l'enseignant est face à la classe... les élèves regardent le tableau... - , d'autre part à interpréter ce qu'ils voient, c'est-à-dire prêter par exemple des intentions à l'enseignant ou aux élèves, faire des hypothèses sur leurs "états mentaux» ou leurs vécus émotionnels. Cet exercice "d'ascèse normative " (répété plusieurs fois) permet aux participants de saisir des pans de l'activité visionnée jusque-là invisibles pour eux, et aussi de se préparer à regarder de manière fine et contrastée leur propre activité. À partir de ce double travail de description et d'interprétation, l'activité visionnée peut être ré-évaluée, ceci non pas dans une optique de "manque" ou de "défaut", mais en y cherchant les dimensions positives non encore suffisamment développées, mais déjà-là «en germe ".

Ce travail pratique peut être soutenu par la présentation de couples de concepts classiques issus du champ de l'analyse du travail - travail prescrit vs travail réel, activité réalisée vs activité réelle, activité productive vs activité constructive -, et aussi prolongé dans des exercices pratiques d'initiation à certaines méthodes d'analyse de l'activité - autoconfrontation simple, croisée, instruction au sosie,... - mais portant sur des objets «sans enjeux » : par exemple, on demande à une personne d'apprendre à faire un nœud compliqué, on la filme et on l'autoconfronte. 


\section{Ouverture temporelle, objectale et relationnelle}

On peut décrire "idéellement" le déroulement temporel linéaire d'une enquête collaborative dans laquelle sont engagés un collectif d'enseignants et une équipe de chercheurs-accompagnateurs. Le premiers temps est celui de la demande de mise sur pied d'une enquête collaborative, demande qui peut être formulée par le collectif d'enseignants lui-même, mais qui, le plus souvent, est formulée par d'autres instances « hiérarchiquement» supérieures. Ce moment correspond bien à ce que Dewey appelle une situation indéterminée (trouble, opaque, douteuse...). Lorsque la demande ne vient pas du collectif enseignant lui-même, le «sentiment » d'indétermination ainsi que le besoin lié de transformation de la situation ne sont pas toujours au rendez-vous.

Le second temps est celui d'une double négociation : négociation concernant le(s) objet(s) d'enquête (co-construction inter-objective), mais négociation aussi des modes d'interventions des chercheurs-accompagnateurs ainsi que des modes d'engagement des acteurs, et ceci principalement en ce qui concerne le recueil de traces de l'activité (activité filmée ou pas, nombre d'interventions, type de travail sur les traces recueillies, etc.). Si ce double moment de négociation est important quelle que soit l'origine de la demande c'est lui qui fonde la nature collaborative de l'enquête -, il l'est d'autant plus quand cette dernière n'est pas venue du collectif lui-même : d'une certaine manière la co-construction d'objets d'enquête consiste aussi en une construction de demande du collectif. Ce deuxième moment correspond souvent à la double "production" d'une situation indéterminée suivie d'une situation problématique. C'est aussi le moment où se joue l'élaboration d'un « simple » collectif en une communauté.

6 Le troisième temps de l'enquête va consister à recueillir des traces de l'activité, à confronter individuellement ou collectivement les acteurs à ces traces, ainsi à mener un double travail de meilleure compréhension de leur activité (relativement bien sûr aux objets d'enquête) et de formulation d'hypothèses pratiques de transformation majorante de celle-ci. Ces hypothèses pratiques seront mises en œuvre, susciteront de nouveaux recueil de traces, sur lesquelles sera à nouveau mené un double travail d'analyse et de formulation d'hypothèses pratiques... et ainsi de suite, jusqu'à ce que le collectif arrive à la conclusion que le problème initialement identifié a trouvé une solution satisfaisante, bref qu'on a affaire à une nouvelle situation déterminée.

Dans les faits, les choses sont beaucoup plus enchevêtrées, voire embrouillées, car objets, temporalité et place des différents acteurs de l'enquête restent à tout moment " ouverts " et ceci solidairement. Une ouverture temporelle d'abord, car si on peut (plus ou moins) dire quand l'enquête a commencé, on ne sait jamais vraiment, ni quand elle va se clore, ni même «à quel stade » on se trouve à tel ou tel moment. En effet, les objets d'enquête sont " poreux » à la dimension négociatrice de l'enquête : ils tendent à se transformer, à se déplacer... Pour le dire autrement, les problèmes soulevés ne s'achèvent pas tant dans des solutions qui leurs sont trouvées que dans la mise à jour de nouveaux problèmes. Les situations problématiques ne se reconfigurent pas tant dans des situation déterminées que dans de nouvelles situations indéterminées.

De plus, cette ouverture temporelle et objectale ne se déploie pas « d'un bloc », les acteurs engagés dans l'enquête ne s'y "déplaçant » pas tous à la même vitesse : des objets encore d'actualité pour certains acteurs sont jugés obsolètes par d'autres. Ce qui suppose aussi des transformations concernant tant le mode d'engagement des acteurs dans l'enquête 
(ce qu'ils y font, ce qu'ils y apportent) que la force de cet engagement (le degré de leurs apports) : certains qui exercent une sorte de leadership à un certain moment peuvent se retirer dans une position plus d'attente à un autre.

\section{Un exemple de développement « ouvert » d'une enquête collaborative}

Le projet Plus de maitres que de classes concrétisant un partenariat entre l'Université de Genève et le Département de la Haute-Savoie a été négocié à un triple niveau :

1) entre l'inspection départementale de l'Education Nationale et les chercheurs, afin d'élaborer un partenariat contractualisé délimitant les objectifs poursuivis, la temporalité et les moyens mis en œuvre par chacun des partenaires ;

2) avec les équipes de circonscription (conseillers pédagogiques et inspecteurs qui ont en charge la formation et l'évaluation des personnels enseignants), afin de définir les modalités d'engagement de ces partenaires (dans le groupe de pilotage et/ou dans la mise en œuvre dans les écoles);

3) avec les équipes enseignantes des écoles (directeur, maître supplémentaire, autres maîtres) relativement aux modalités de recherche, au recueil de traces d'activité (parfois filmées) et à l'accompagnement à la mise en œuvre d'enquêtes collectives dans les écoles.

A chaque niveau, une nouvelle négociation a dû avoir lieu pour que l'objet d'enquête soit réaménagé au plus près des préoccupations de chacun des protagonistes impliqués.

Pour l'inspection, l'objet était le développement de nouvelles modalités d'accompagnement du développement professionnel des enseignants et des conseillers pédagogiques à travers l'usage de la vidéo et de l'enquête dans les écoles, ceci dans une perspective d'innovation et d'amélioration des pratiques pédagogiques, puis, par extension, des apprentissages des élèves.

Pour les conseillers pédagogiques, la préoccupation était de s'approprier une nouvelle professionnalité de formateur «accompagnant» le développement professionnel des enseignants, plutôt que « transmettant » des savoirs préétablis.

Enfin, pour les enseignants, les préoccupations visaient d'abord une meilleure compréhension $\mathrm{du}$ «bien fonctionner à deux dans les classes", ensuite la "conjugaison féconde » de leurs interventions pour mieux faire apprendre les élèves.

Dans les trois écoles où des enquêtes ont été mises sur pied, la focalisation sur l'activité des enseignants et le fonctionnement à deux présente la première année a évolué en direction d'une meilleure compréhension de l'activité des élèves les années suivantes.

Tout ceci ne signifie pas que ce type d'enquête soit condamné à tourner en rond, à bégayer, ou à se ramifier jusqu'à l'éclatement. Cette triple ouverture, ainsi que les tensions et les incertitudes qui vont avec - et qui sont à « contenir » et non à éliminer -, sont la marque que la «nature» inter-objective et négociatrice de l'enquête reste au cœur de son déploiement.

\section{Des enquêtes soutenues par une rationalité pratique}

Les raisonnements, les argumentations, les contenus des controverses déployés dans les enquêtes collaboratives procèdent d'une rationalité pratique et située, dont on peut décrire quelques-unes des caractéristiques principales. 
51 Premièrement, la rationalité des acteurs répond à un engagement fort, intime, dans leur travail. Les travaux d'ergologie (Schwartz, 2009) et de clinique de l'activité (Clot, 2008) ont montré que le travail suscite des mises à l'épreuve et des investissements subjectifs qui vont bien au-delà d'une simple réponse objective à ce qui est demandé. Ces mises à l'épreuve de la subjectivité sont encore renforcées lorsqu'il s'agit de transformer le travail.

Ensuite, les enquêtes collaboratives portent autant sur des valeurs que sur des faits ${ }^{7}$ et les énoncés utilisés par les acteurs pour parler ou débattre de leur activité font un usage important de ce que Putnam (2004) appelle des concepts éthiques épais ${ }^{8}$, c'est-à-dire simultanément descriptifs et évaluatifs (pensons par exemple à l'élève lent, où à l'inverse rapide, ou déconcentré...). En conséquence, les évaluations que les acteurs font de leur action « débordent " largement la mesure des résultats de celle-ci. Ils tendent à faire des évaluations fortes (Taylor, 1977) de leur action - c'est-à-dire portant des enjeux identitaires - relatives à ce qu'ils croient qu'ils devraient être, ou à ce qu'ils cherchent à être comme personnes. ${ }^{9}$

53 À partir de là, les débats et controverses menés dans le cadre des enquêtes collaboratives s'appuient sur une rationalité expressive (Cavell, 1996 ; 2009) - c'est-à-dire se constituant dans le travail d'expression du « monde » des normes, des valeurs, des significations dans lequel les acteurs collectifs et individuels sont profondément engagés - et transitionnelle (Taylor, 1993, 2003) - c'est-à-dire ancrée dans des processus d'expression, de comparaison et d'articulation de positions divergentes, ainsi qu'à la recherche de solutions situées, provisoires et communes.

Un exemple de déploiement de la rationalité pratique dans une enquête collaborative

Lors des enquêtes collectives menées avec des équipes enseignantes du projet Plus de maîtres que de classes nous avons pu constater à quel point les concepts utilisés par les enseignants, quand ils parlent de leurs élèves, sont de type épais et les autorisent simultanément à décrire et évaluer leurs comportements : ils les disent motivés, dissipés, à niveau, lents, timides...

Ces enquêtes sont loin de porter uniquement sur des "résultats objectifs" comme, par exemple, les apprentissages des élèves. Très souvent, c'est ce que les enseignants pensent que leur travail devrait être, voire ce qu'ils pensent devoir être (posture, attitude...) qui est jugé, voire remis en question. Il s'agit de savoir si moi, en tant que personne, je fais bien mon travail ou pas : on a ainsi affaire à des évaluations fortes.

Par exemple, une équipe d'enseignants visant à faire travailler les élèves « en autonomie " s'est posée la question de la redéfinition de leur rôle dans ce cadre. Cette redéfinition, dans la mesure où elle impliquait des moments de suspension dans les interventions, a amené les enseignants à se demander s'ils faisaient toujours bien leur travail, si c'était bien cela qui leur était demandé, s'ils n'étaient pas en train de se trahir ou de trahir leurs élèves....

L'enquête a ainsi achoppé sur deux postures divergentes impliquant des évaluations fortes, des conflits de normes et de valeurs: d'un côté les enseignants qui ne conçoivent pas que l'apprentissage chez les élèves puisse avoir lieu en dehors de l'interaction avec l'enseignant et qui considèrent donc le travail en autonomie comme une activité "par défaut", occupationnelle, visant uniquement à dégager un espace privilégié d'interaction enseignantélève; de l'autre, ceux pour qui la capacité de l'élève de travailler en autonomie est une des compétences principales que l'école doit développer ainsi qu'une des modalités premières dans laquelle l'élève apprend et qui soignent tout particulièrement la préparation des activités qui doivent être effectuées par l'élève durant ce temps de travail. 


\section{Conclusion}

$\mathrm{Au}$ terme de cet article, nous aimerions souligner le statut encore exploratoire de l'usage de ces enquêtes dans la formation initiale et continue des enseignants. Ces dernières ouvrent en effet autant de pistes prometteuses qu'elles suscitent de nouvelles questions. Elles représentent toutefois des outils pertinents et innovants pour penser la formation dans une dimension " processus » en complémentarité avec les dimensions "produits » (savoirs, compétences) plus portées par les dispositifs traditionnels. Il s'agit maintenant d'observer et de documenter si et comment les processus d'enquête inter-objective conceptuellement décrits ici s'opérationnalisent dans les formations. Comment les formateurs-accompagnateurs peuvent-ils configurer au mieux des environnements « augmentés » pour susciter ces processus ? Comment les chercheurs sont-ils capables de rendre compte des transformations d'activités générées par ces processus?

\section{BIBLIOGRAPHIE}

Cavell, S. (1996). Les voix de la raison. Paris : Seuil.

Cavell, S. (2009). Dire et vouloir dire. Paris : Seuil.

Clot, Y. (2008). Travail et pouvoir d'agir. Paris : PUF.

Descombes, V. (2004). Le complément de sujet. Enquête sur le fait d'agir de soi-même. Paris : Gallimard.

Dewey, J. (1911). Contributions at A Cyclopedia of Education, MW, vol. 6. Carbondale : Southern Illinois University Press.

Dewey, J. (1993). Logique. Théorie de l'enquête. Paris : PUF.

Dewey, J. (2010). Le public et ses problèmes. Paris : Folio.

Dewey, J. (2011). La formation des valeurs. Paris : La Découverte.

Flandin, S., Leblanc, S. \& Muller, A. (2015). Vidéoformation « orientée activité » : quelles utilisations pour quels effets sur les enseignants? In M. Durand, V. Lussi Borer \& F. Yvon (Eds.), Analyse du travail et formation dans les métiers de l'éducation (pp. 179-198). Bruxelles : De Boeck.

Lussi Borer, V. \& Muller, A. (2013, avril). Analyse de l'activité, environnements de formation et développement professionel. Différences intra et inter-activités : le possible, le réel et le virtuel. Texte présenté au Colloque international en éducation « Enjeux actuels et futurs de la formation et de la profession enseignante » organisé par le CRIFPE, Montréal.

Lussi Borer, V., \& Muller, A. (2014a). Exploiter le potentiel des processus de renormalisation en formation à l'enseignement. @ctivités, 11(2), 129-142.

Lussi Borer, V., \& Muller, A. (2014b). Connaître l'activité des enseignants en formation sur la plateforme Néopass@ction. Recherche et Formation, 75, 65-80. 
Lussi Borer, V., \& Muller, A. (2016). L'enquête collaborative comme démarche de transformation de l'activité d'enseignement : de la formation initiale à la formation continuée. In L. Ria \& V. Lussi Borer (Eds), Apprendre à enseigner. Paris : PUF.

Muller, A. (2014). Analyse de l'activité dans la formation des enseignants secondaires. Educateur, 05/2014, 15-16.

Muller, A., \& Lussi Borer, V. (2017). Initier une enquête collaborative orientée activité : travail du formateur et des formés. Travail et Apprentissage, 17, 58-80.

Peirce, C.S. (1978). Écrits sur le signe. Paris : Seuil.

Putnam, H. (2004). Fait/Valeur : la fin d'un dogme et autres essais. Paris-Tel-Aviv : Editions de l'Eclat.

Putnam, H. (2013). L'Ethique sans l'Ontologie. Paris : Les Editions du Cerf.

Schwartz, Y. (2009). Dialogue 1. Trajectoires et usages de soi. In Y. Schwartz \& L. Durrive (Eds.), L'Activité en dialogues. Entretiens sur l'activité humaine (II) (pp. 9-33). Toulouse : Octarès.

Taylor, C. (1977). What is Human Agency? In T. Mischel (Ed.), The Self (pp. 103-135). Oxford: Blackwell.

Taylor, C. (1993). Explanation and Practical Reason. In M. Nussbaum \& A. Sen (Eds.), The Quality of Life (pp. 208-231). Oxford : Clarendon.

Taylor, C. (2003). Les sources du moi. Paris : Seuil.

Zask, J. (2004). L'enquête sociale comme inter-objectivation. In B. Karsenti \& L. Quéré (Eds.), La croyance et l'enquête. Aux sources du pragmatisme (pp. 141-163). Bruxelles : De Boeck.

Zask, J. (2015). Introduction à John Dewey. Paris : La Découverte.

\section{NOTES}

1. Et c'est dans ce sens qu'il faudra comprendre dans ce texte certaines expressions qui pourraient être perçues comme renvoyant à une sorte de "vécu intérieur subjectif »: par exemple point de vue, interprétation, perception, émotions... Ces expressions renvoient, non pas à un "vécu intérieur", mais à des fonctions différentes du processus de transaction organisme environnement, une certaine modalité de leur relation. De même parler de sujet, renvoie à un des pôles de la relation qui se saisit comme je, comme agent (Descombes, 2004) dans le cadre de celleci. Si ces expressions tendent à «sonner » subjectives - point de vue subjectif, interprétation subjective, perception subjective, etc. - c'est, qu'au-delà même d'une tradition épistémologique qui nous entraîne à voir les choses comme cela, notre langage ordinaire repose logiquement sur la mise en relation d'entités premières - sujet et objet par exemple - qui sont mises en relation et non pas sur une relation première constitutive de ces termes. Par là-même, ce qui d'un point de vue transactionnel qualifie un certain mode de relation est «tout naturellement " versé du côté de l'organisme ou du côté de l'environnement.

2. Ces processus (et d'autres) ont été identifiés dans toute une série de recherches dont on trouve la synthèse dans Flandin, Leblanc \& Muller (2015).

3. Pour un compte-rendu empirique de ce type de processus, voir aussi Muller (2014).

4. Pour un compte-rendu empirique de ce type de processus, voir aussi Lussi Borer \& Muller (2013).

5. Les mêmes phénomènes se produisent dans la confrontation à des traces de l'activité d'autrui. 
6. Nous parlons d'hypothèse car il nous semble impossible - ou pour le moins non réalisable pour le moment eu égard aux ressources méthodologiques disponibles - d'identifier des données empiriques témoignant d'un tel degré de complexité interactionnelle.

7. Dewey $(2011)$ et plus tard Putnam $(2004,2013)$ ont totalement remis en question cette dichotomie, du moins quand elle prend une dimension métaphysique.

8. Les concepts éthiques fins, sont ceux qui sont uniquement évaluatifs : par exemple : bon, bien, mal...

9. Les évaluations faibles portent, elles, uniquement sur les résultats d'une action, et sont détachées des personnes.

\section{RÉSUMÉS}

Cet article décrit et illustre comment nous utilisons les concepts d'enquête inter-objective et de transaction entre organisme et environnement développés par Dewey pour mettre sur pied et expérimenter des d'environnements "augmentés" dans le cadre de formations initiale et continue à l'enseignement. Les enquêtes menées sur ces environnements " augmentés » à partir de traces d'activité filmées et d'expérience du travail visent à permettre aux participants à l'enquête de transformer leur activité ainsi qu'à se développer professionnellement. L'article explore les différentes transactions que le participant peut expérimenter/vivre lorsqu'il est confronté aux traces de son activité, à l'inter-objectivation de sa propre activité durant l'enquête, ainsi que les espaces de nouvelles significations qui peuvent dès lors s'ouvrir. Enfin, il décrit les principes, les modalités et les conditions d'opérationnalisation qui structurent de tels environnements de formation ainsi que les tensions que les formateurs rencontrent lors de leur mise en œuvre étant donné la structure ouverte de l'enquête collaborative et la rationalité pratique qu'elle implique.

This article describes and illustrates how we use the concepts of collaborative inquiry and transaction between organism and environment developed by Dewey to design and experiment video-enhanced learning environments in teacher education and professional development programs. Inquiries conducted on these video-enhanced learning environments are based on traces of filmed activity and experience of work. It aims to enable the participants to the inquiry to transform their teaching activity and develop professionally. The article explores the different transactions that participants can experience when confronted with the traces of their activity, the inter-objectification of their own activity during the inquiry, and spaces of new meanings that are created. Finally, it describes the principles, modalities and conditions of operationalization that structure such video-enhanced environments and the tensions that facilitators encounter during the program, given the open structure of the collaborative inquiry and the practical rationality it implies.

\section{INDEX}

Keywords : enquête inter-objective, transaction organisme-environnement, environnement « augmenté », formation des enseignants, développement professionnel 


\section{AUTEURS}

\section{ALAIN MULLER}

Chargé d'enseignement à l'Institut Universitaire de Formation des Enseignants de l'Université de Genève et membre des équipes de recherche CRAFT et LIFE

\section{VALÉRIE LUSSI BORER}

Maître d'enseignement et de recherche à l'Institut Universitaire de Formation des Enseignants et à la Faculté de psychologie et des sciences de l'éducation de l'Université de Genève (Suisse), membre de l'équipe de recherche CRAFT. 